\title{
SOME MATRIX INEQUALITIES OF KY FAN TYPE
}

\author{
B. MOND AND J. E. PEC̆ARIĆ
}

\begin{abstract}
One of the well-known generaliztions of the Kantorovich inequality was given by Ky Fan who established his results for one positive definite matrix but for several vectors. Here we give corresponding results for several matrices and vectors. As well, a number of related inequalities are established.
\end{abstract}

\section{Introduction}

Ky Fan [3] proved the following result: Let $A$ be a positive definite Hermitian matrix of order $n$ with all its eigenvalues contained in the closed interval $[a, b]$, where $0<a<b$. Let $x_{1}, \ldots, x_{k}$ be any finite number of vectors in the unitary $n$-space such that $\sum_{j=1}^{k}\left\|x_{j}\right\|^{2}=1$. Then, for every integer $p \neq 0,1$ (not necessarily positive), we have

$$
\frac{\sum_{j=1}^{k}\left(A^{p} x_{j}, x_{j}\right)}{\left[\sum_{j=1}^{k}\left(A x_{j}, x_{j}\right)\right]^{p}} \leq \frac{(p-1)^{p-1}}{p^{p}} \frac{\left(b^{p}-a^{p}\right)^{p}}{(b-a)\left(a b^{p}-b a^{p}\right)^{p-1}} .
$$

In particular, the following generalization of the Kantorovich-Hermite inequality holds

$$
\left[\sum_{j=1}^{k}\left(A x_{j}, x_{j}\right)\right]\left[\sum_{j=1}^{k}\left(A^{-1} x_{j}, x_{j}\right)\right] \leq \frac{(a+b)^{2}}{4 a b}
$$

In this paper, we give similar results with several matrices, for these as well as various related inequalities.

\section{Preliminaries}

Let $A \in C^{n \times n}$ be a normal matrix, i.e., $A^{*} A=A A^{*}$. Here $A^{*}$ means $\bar{A}^{t}$, the transpose conjugate of $A$. There exists [4] a unitary matrix $U$ such that

$$
A=U^{*}\left[\lambda_{1}, \lambda_{2}, \ldots, \lambda_{n}\right] U
$$

Received March 23, 1994; revised June 15, 1994.

1991 Mathematics Subject Classification. 15A45.

Key words and phrases. Kantorovich inequality, ratio of means, differences of means. 
where $\left[\lambda_{1}, \ldots, \lambda_{n}\right]$ is the diagonal matrix $\left(\lambda_{j} \delta_{i j}\right)$, and where $\lambda_{1}, \lambda_{2}, \ldots, \lambda_{n}$ are the eigenvalues of $A$, each appearing as often as its multiplicity. $A$ is Hermitian if and only if $\lambda_{i}$, $i \in I_{n}=\{1,2, \ldots, n\}$ are real. If $A$ is Hermitian and all $\lambda_{i}$ are strictly positive, then $A$ is said to be positive definite. Assume now that $f\left(\lambda_{i}\right) \in C, i \in I_{n}$ is well defined. Then $f(A)$ may be defined by (see e.g. [4, p.71] or $[1$, p.90])

$$
f(A)=U^{*}\left[f\left(\lambda_{1}\right), f\left(\lambda_{2}\right), \ldots, f\left(\lambda_{n}\right)\right] U
$$

As before, if $f\left(\lambda_{i}\right), i \in I_{n}$ are all real, then $f(A)$ is Hermitian. If, also, $f\left(\lambda_{i}\right)>0, i \in I_{n}$, then $f(A)$ is positive definite.

We note that for the inner product

$$
(f(A) x, x)=\sum_{i=1}^{n}\left|y_{i}\right|^{2} f\left(\lambda_{i}\right)
$$

where $y \in C^{n}, y=U x, x=\left(x_{1}, \ldots, x_{n}\right) \in C^{n}$, and so $\sum_{i=1}^{n}\left|y_{i}\right|^{2}=\sum_{i=1}^{n}\left|x_{i}\right|^{2}$.

If $A$ is positive definite, so that $\lambda_{i}>0, i \in I_{n}$ and $f(t)=t^{r}$ where $t>0$ and $r \in R$, we have $f(A)=A^{r}$.

Let $S$ be the set of all $j, i ; j \in\{1, \ldots, k\}, i \in\{1, \ldots, n\}$ such that $y_{j i} \neq 0$ where $y_{j}=U_{j} x_{j}$

\section{Inequalities for Convex Functions}

Theorem 1. Let $f$ be a convex function on $I=[m, M] \subset R$ and $A_{j}, j=$ $1, \ldots, k$, be Hermitian matrices with eigenvalues $\lambda_{j i}(i=1,2, \ldots, n)$ in $I ; x_{j} \in C^{n}$, $j=1, \ldots, k$ with $\sum_{j=1}^{k}\left(x_{j}, x_{j}\right)=1$. Then

$$
\sum_{j=1}^{k}\left(f\left(A_{j}\right) x_{j}, x_{j}\right) \leq \frac{M-\bar{x}}{M-m} f(m)+\frac{\bar{x}-m}{M-m} f(M)
$$

where

$$
\bar{x}=\sum_{j=1}^{k}\left(A_{j} x_{j}, x_{j}\right) .
$$

If $f$ is strictly convex, equality holds in (6) if and only if, for all $(j, i) \in S, \lambda_{j i}=m$ or $\lambda_{j i}=M$.

Proof. First we note that $\sum_{j=1}^{k} \sum_{i=1}^{n}\left|y_{j i}\right|^{2}=\sum_{j=1}^{k}\left(x_{j}, x_{j}\right)=1$. We now have, by

$$
\bar{x}=\sum_{j=1}^{k}\left(A_{j} x_{j}, x_{j}\right)=\sum_{j=1}^{k} \sum_{i=1}^{n}\left|y_{j i}\right|^{2} \lambda_{j i}
$$




$$
\sum_{j=1}^{k}\left(f\left(A_{j}\right) x_{j}, x_{j}\right)=\sum_{j=1}^{k} \sum_{i=1}^{n}\left|y_{j i}\right|^{2} f\left(\lambda_{j i}\right)
$$

Further, by the defining inequality for convex functions, we have

$$
f\left(\lambda_{j i}\right) \leq \frac{M-\lambda_{j i}}{M-m} f(m)+\frac{\lambda_{j i}-m}{M-m} f(M)
$$

Multiplying (9) by $\left|y_{j i}\right|^{2}$ and summing, we get

$$
\begin{aligned}
& \sum_{j=1}^{k} \sum_{i=1}^{n}\left|y_{j i}\right|^{2} f\left(\lambda_{j i}\right) \\
\leq & \frac{1}{M-m}\left\{\left(M-\sum_{j=1}^{k} \sum_{i=1}^{n}\left|y_{j i}\right|^{2} \lambda_{j i}\right) f(m)+\left(\sum_{j=1}^{k} \sum_{i=1}^{n}\left|y_{j i}\right|^{2} \lambda_{j i}-m\right) f(M)\right\}
\end{aligned}
$$

which is, with respect to (8), the same as (6).

Theorem 2. Let $f, A_{j}, \lambda_{j i}, x_{j}$ be as in Theorem $1 ; F: J^{2} \rightarrow R$ be increasing in its first variable, and $J$ an interval containing the range of $f$; then

$$
\begin{aligned}
& F\left\{\sum_{j=1}^{k}\left(f\left(A_{j}\right) x_{j}, x_{j}\right), f\left(\sum_{j=1}^{k}\left(A_{j} x_{j}, x_{j}\right)\right)\right\} \\
\leq & \max _{m \leq t \leq M}\left\{F\left(\frac{M-t}{M-m} f(m)+\frac{t-m}{M-m} f(M), f(t)\right)\right\} \\
= & \max _{\theta \in[0,1]} F(\theta f(m)+(1-\theta) f(M), f(\theta m+(1-\theta) M))
\end{aligned}
$$

Proof. By the increasing character of $F$ and by (6) we have

$$
\begin{aligned}
& F\left\{\sum_{j=1}^{k}\left(f\left(A_{j}\right) x_{j}, x_{j}\right), f\left(\sum_{j=1}^{k}\left(A_{j} x_{j}, x_{j}\right)\right)\right\} \\
\leq & F\left\{\frac{M-\bar{x}}{M-m} f(m)+\frac{\bar{x}-m}{M-m} f(M), f(\bar{x})\right\} \\
\leq & \max _{m \leq t \leq M}\left\{F\left(\frac{M-t}{M-m} f(m)+\frac{t-m}{M-m} f(M), f(t)\right)\right\}
\end{aligned}
$$

since $\bar{x} \in[m, M]$.

Theorem 2 is a generalization of a result given in [5]. Moreover the following result is also a consequence of Theorem 2 (one must take $F(t, s)=t-s$ in Theorem 2). 
Theorem 3. If $f: I \rightarrow R, f(t)>0, f^{\prime \prime}(t)>0$ for $t \in I(=[m, M]) ; A_{j}, j=$ $1, \ldots, k$ are Hermitian matrices with eigenvalues in $I: x_{j} \in C^{n}$ with $\sum_{j=1}^{k}\left(x_{j}, x_{j}\right)=$ $1, j=1, \ldots, k$. Then

$$
\sum_{j=1}^{k}\left(f\left(A_{j}\right) x_{j}, x_{j}\right) \leq \theta+f\left(\sum_{j=1}^{k}\left(A_{j} x_{j}, x_{j}\right)\right)
$$

where $\theta=\mu \phi(\mu)+\gamma-(f \circ \phi)(\mu)$, and $\phi=\left(f^{\prime}\right)^{-1}, \mu=\frac{f(M)-f(m)}{M-m}, \gamma=\frac{M f(m)-m f(M)}{M-m}$.

\section{Inequalities for Power Means}

Let $A_{j}, j=1, \ldots, k$ be positive definite Hermitian matrices with eigenvalues $\lambda_{j 1} \geq$ $\lambda_{j 2} \geq \ldots \geq \lambda_{j n}, r$ a real number, $x_{j} \in C^{n}, j=1, \ldots, k$ with $\sum_{j=1}^{k}\left(x_{j}, x_{j}\right)=1$. Then we can introduce the following power means:

$$
M_{k}^{[r]}(A ; x)= \begin{cases}{\left[\sum_{j=1}^{k}\left(A_{j}^{r} x_{j}, x_{j}\right)\right]^{1 / r},} & r \neq 0 \\ \exp \left[\sum_{j=1}^{k}\left(\left(\log A_{j}\right) x_{j}, x_{j}\right)\right], & r=0\end{cases}
$$

For $r<s$, we have $([5])$ :

$$
M_{k}^{[r]}(A ; x) \leq M_{k}^{[s]}(A ; x)
$$

with equality if and only if $\lambda_{j i},(j, i) \in S$, are all equal.

A consequence of Theorem 1 is the following:

Theorem 4. If $0<r<s$ or $r<s<0$, then

$$
\begin{aligned}
& \left(M^{r}-m^{r}\right) M_{k}^{[s]}(A ; x)^{s}-\left(M^{s}-m^{s}\right) M_{k}^{[r]}(A ; x)^{r} \\
\leq & M^{r} m^{s}-M^{s} m^{r}
\end{aligned}
$$

and the reverse inequality holds for $r<0<s$. If $r=0<s$, then

$$
\begin{aligned}
& \log \frac{M}{m}\left(M_{k}^{[s]}(A ; x)\right)^{s}-\left(M^{s}-m^{s}\right) \log \left(M_{k}^{[0]}(A ; x)\right) \\
\leq & m^{s} \log M-M^{s} \log m
\end{aligned}
$$

Proof. Replacing $A_{j}$ by $A_{j}^{r}$ and taking $f(t)=t^{s / r}$ or $-t^{s / r}$ in Theorem 1 according as $0<r<s$ or $r<s<0$, we get (16). Other cases are proved similarly. difference:

A ratio of such means was considered in [5]. Here we shall give similar results for a

$$
D_{k}^{r, s}(A ; x)=M_{k}^{[s]}(A ; x)-M_{k}^{[r]}(A ; x)
$$


The following can be proved as a consequence of Theorem 4 or as a consequence of Theorem 7 from [2, pp. 206-207].

Theorem 5. Let $A_{j}$ be positive definite Hermitian matrices with eigenvalues in $[m, M]$. Set $\gamma=M / m$ and let $r$ and $s$ be real munbers, $r<s$. Then

$$
D_{k}^{r, s}(A ; x) \leq \Delta
$$

where $\Delta$ is defined by

$$
\begin{aligned}
\Delta & =\left[\theta M^{s}+(1-\theta) m^{s}\right]^{1 / s}-\left[\theta M^{r}+(1-\theta) m^{r}\right]^{1 / r}, \quad \text { if } \quad r s \neq 0 \\
& =\left[\theta M^{s}+(1-\theta) m^{s}\right]^{1 / s}-M^{\theta} m^{(1-\theta)}, \quad \text { if } \quad r=0 \\
& =M^{\theta} m^{(1-\theta)}-\left[\theta M^{r}+(1-\theta) m^{r}\right]^{1 / r}, \quad \text { if } s=0,
\end{aligned}
$$

and $\theta$ is defined as follow. Let

$$
\begin{array}{cc}
h(x) \equiv x^{1 / s}-(a x+b)^{1 / r} \quad \text { with } & a=\frac{M^{r}-m^{r}}{M^{s}-m^{s}}, \\
b=\frac{M^{s} m^{r}-M^{r} m^{s}}{M^{s}-m^{s}}, \quad \text { if } \quad r s \neq 0 & r s=0 \\
\equiv x^{1 / s}-m \gamma^{\left(x-m^{s}\right) /\left(M^{s}-m^{s}\right)} \quad \text { if } \quad r=0 & \quad \text { if } \quad s=0 .
\end{array}
$$

Let $J$ denote the open interval joining $m^{s}$ to $M^{s}$ if $s \neq 0$, and let $J=\left(M^{r}, m^{r}\right)$ if $s=0$. There is an $x^{*} \in J$ such that $h(x)<h\left(x^{*}\right)$ for every $x\left(\in J, \neq x^{*}\right)$. (Observe that if $r s \neq 0$, then $a x+b>0$ at the end points of $J$ and, therefore, throughout J.) We set

$$
\theta=\left(x^{*}-m^{s}\right) /\left(M^{s}-m^{s}\right) \quad \text { if } \quad s \neq 0
$$

and

$$
\theta=\left(x^{*}-m^{r}\right) /\left(M^{r}-m^{r}\right) \quad \text { if } \quad s=0 .
$$

Proof. Let $0<r<s$. Note that (16) can be written in the following form

$$
M_{k}^{[r]}(A ; x)^{r} \geq a M_{k}^{[s]}(A ; x)^{s}+b
$$

So we have

$$
\begin{aligned}
M_{k}^{[s]}(A ; x)-M_{k}^{[r]}(A ; x) & \leq M_{k}^{[s]}(A ; x)-\left(a M_{k}^{[s]}(A ; x)^{s}+b\right)^{1 / r} \\
=h\left(M_{k}^{[s]}(A ; x)^{s}\right) & \leq h\left(x^{*}\right)
\end{aligned}
$$

The other cases can be proved similarly. 
Remark. Of particular interest are the following special cases of (19):

$$
\sum_{j=1}^{k}\left(A_{j} x_{j}, x_{j}\right)-\left[\sum_{j=1}^{k}\left(A_{j}^{-1} x_{j}, x_{j}\right)\right]^{-1} \leq(\sqrt{M}-\sqrt{m})^{2}
$$

and

$$
\left[\sum_{j=1}^{k}\left(A_{j}^{2} x_{j}, x_{j}\right)\right]^{1 / 2}-\sum_{j=1}^{k}\left(A_{j} x_{j}, x_{j}\right) \leq \frac{(M-m)^{2}}{4(M+m)}
$$

\section{References}

[1] R. Bellman, Introduction to Matrix Analysis, McGraw-Hill, New York, 1960.

[2] P. S. Bullen, D. S. Mitrinović and P. M. Vasić, Means and their inequalities, D. Reidel, Dordrecht/Boston/Lancaster/Tokyo, 1988.

[3] Ky Fan, "Some Matrix Inequalities," Math. Sem. Univ. Hamburg, 29, 185-196, 1966.

[4] M. Marcus and H. Minc, A Survey of Matrix Theory and Inequalities, Allyn and Bacon, Boston, 1964.

[5] B. Mond and J. E. Pečarić, "Generalization of a matrix inequality of Ky Fan," J. Math. Anal. Appl., 190, 244-247, 1995.

[6] J. E. Pečarić and P. R. Beesack, "On Knopp's inequality for convex functions," Canad. Math. Bull., 30, 267-272, 1987.

School of Mathematics, La Trobe University, Bundoora, Victoria, 3083, Australia.

Faculty of Textile Technology, University of Zagreb, Zagreb, Croatia. 